\title{
Renal Artery
}

National Cancer Institute

\section{Source}

National Cancer Institute. Renal Artery. NCI Thesaurus. Code C12778.

Either of two arteries (right or left) that arise from the aorta to supply the kidneys. 